\title{
ON CREATIVE SETS AND INDICES OF PARTIAL RECURSIVE FUNCTIONS
}

\author{
BY \\ LOUISE HAY(1)
}

1. Introduction. Creative sets were introduced by Post [7] in the course of his investigation of the degrees of unsolvability of the decision problems of recursively enumerable (r.e.) sets of positive integers. This investigation was largely motivated by the correspondence, under a Gödel numbering, between r.e. sets and the sets of provable statements of recursively axiomatizable formal systems. Under this interpretation, creativity of a set corresponds to effective undecidability of the system under consideration, a viewpoint further developed in the work of Myhill [5] and others. In [10], Smullyan generalized these notions by introducing double creativity of pairs of r.e. sets, which corresponds to effective inseparability of the sets of provable and refutable statements ( $T$ and $R$ respectively) of recursively axiomatizable theories. In this paper, we relate these notions to sets of indices of partial recursive functions (i.e., sets $\theta f=\left\{n \mid q_{n} \simeq f\right\}$ ). Our main result is that double creativity of a pair $(\alpha, \beta)$ of disjoint r.e. sets is equivalent to separation by $\alpha$ and $\beta$ of a pair of sets isomorphic to some pair $(\theta f, \theta g)$ under a recursive permutation. "Relativization" of this result to formal theories yields the existence of effectively inseparable sets of statements other than Smullyan's "nuclei" $T$ and $R$.

2. Effective inseparability of sets of indices of partial recursive functions. Let $\theta q_{a}=\left\{n \mid q_{n} \simeq q_{a}\right\}$ in a Kleene enumeration of partial recursive functions. Let $w_{a}=$ range $q_{a}$ and $\theta w_{a}=\left\{n \mid w_{n}=w_{a}\right\}$. Both the null function and the null set will be denoted by $\varnothing$.

DEFINITION 1. A recursive function $F(x, y)$ will be called doubly productive for a pair of sets $(\alpha, \beta)$ if, whenever $w_{i} \subseteq \alpha$ and $w_{j} \subseteq \beta$, then $F(i, j) \in \alpha \cap \beta$ $-\left(w_{i} \cup w_{j}\right)$.

THEOREM 1. There is a recursive function $F$ of four variables such that if $q_{a}, q_{b}$ are partial recursive functions with $q_{a} \neq q_{b}, q_{a} \neq \varnothing, q_{b} \neq \varnothing$, then $F(a, b, x, y)$ is doubly productive for the pair $\left(\theta q_{a}^{\prime}, \theta q_{b}^{\prime}\right)$.

Proof. Define $F(a, b, i, j)$ according to the following effective procedure: first define a partial recursive function $G$ of two variables by

Presented to the Society, January 23, 1965 under the title Effective inseparability of sets of indices of partial recursive functions; received by the editors January 23, 1965 and, in revised form, July 29, 1965.

(1) This paper is a portion of the author's doctoral dissertation written at Cornell University. 
(1) $G(y, x) \simeq q_{b}(x)$ if $(E u)\left[y=q_{j}(u) \wedge(v)_{v \leqq u} y \neq q_{i}(v)\right]$,

(2) $G(y, x) \simeq q_{a}(x)$ if $(E u)\left[y=q_{i}(u) \wedge(v)_{v<u} y \neq q_{j}(v)\right]$,

(3) $G(y, x)$ undefined if $\neg(E u)\left[y=q_{i}(u) \vee y=q_{j}(u)\right]$.

The Gödel number of $G$ is clearly a recursive function of $a, b, i, j$. Now by Kleene's second recursion theorem, there is a number $e$, which can be effectively computed from the Gödel number of $G$ and thus from $a, b, i, j$, such that

(4) $q_{e}(x) \simeq G(e, x)$.

Then set $F(a, b, i, j)=e$.

Now assume that $w_{i} \subseteq \theta q_{a}^{\prime}, w_{j} \subseteq \theta q_{b}^{\prime}$. We claim that $e \in\left(\theta q_{a} \cup \theta q_{b} \cup w_{i} \cup w_{j}\right)^{\prime}$. Assume not:

Case 1. $e \in w_{i} \cup w_{j}$. Define a recursive enumeration of the set $w_{i} \cup w_{j}$ by means of the partial recursive function $f$ defined by $f(2 n)=q_{i}(n), f(2 n+1)$ $=q_{j}(n), n=0,1, \cdots$. Now $(E t)(e=f(t))$, and let $t^{\prime}$ be the least such $t$.

Subcase 1.1. $t^{\prime}$ is even, say $t^{\prime}=2 u^{\prime}$. Then $v<u^{\prime}$ implies $2 v+1<2 u^{\prime}=t^{\prime}$, so that

i.e.,

$$
(E u)\left[e=f(2 u) \wedge(v)_{v<u} e \neq f(2 v+1)\right]
$$

$$
(E u)\left[e=q_{i}(u) \wedge(v)_{v<{ }_{u} e} e \neq q_{j}(v)\right] .
$$

Then by (2) and (4), $q_{e}(x) \simeq G(e, x) \simeq q_{a}(x)$, so that $e \in \theta q_{a}$. But $e=q_{i}(u)$ implies $e \in w_{i} \simeq \theta q_{a}^{\prime}$, which is a contradiction.

Subcase 1.2. $t^{\prime}$ is odd, say $t^{\prime}=2 u^{\prime}+1$. Then $v \leqq u^{\prime}$ implies $2 v<2 u^{\prime}+1=t^{\prime}$, so that $(E u)\left[e=q_{j}(u) \wedge(v)_{v \leqq u} e \neq q_{i}(v)\right]$. Then by (1) and (4),

$$
q_{e}(x) \simeq G(e, x) \simeq q_{b}(x),
$$

so that $e \in \theta q_{b}$. But $e=q_{j}(u)$ implies $e \in w_{j} \subseteq \theta q_{b}^{\prime}$, which again yields a contradiction.

Case 2. $e \in\left(\theta q_{a} \cup \theta q_{b}\right)-\left(w_{i} \cup w_{j}\right)$. Then $e \in\left(w_{i} \cup w_{j}\right)^{\prime}$, so that by (3), $G(e, x)$ is undefined for all $x$, i.e., $q_{e}(x) \simeq G(e, x) \simeq \varnothing$. But $e \in \theta q_{a}$ implies $q_{e}(x) \simeq q_{a}(x)$, while $e \in \theta q_{b}$ implies $q_{e}(x) \simeq q_{b}(x)$. In either case, the hypothesis that $q_{a} \neq \varnothing$ $q_{b} \neq \varnothing$ is contradicted.

Note that the hypothesis of Theorem 1 cannot be weakened to allow $q_{a}$ or $q_{b}$ to be null: if, say, $q_{a}=\varnothing$, then $\theta q_{a}^{\prime}=(\theta \varnothing)^{\prime}$ is r.e., and if we let

$$
w_{i}=\theta q_{a}^{\prime}, w_{j}=\varnothing \subseteq \theta q_{b}^{\prime}
$$

we cannot have $F(a, b, i, j) \in\left(\theta q_{a} \cup \theta q_{b} \cup \theta q_{a}^{\prime} \cup \varnothing\right)^{\prime}=\varnothing$; thus the, conclusion or the theorem fails to hold for any $F$.

COROLlaRY 1.1. If $f, g$ are distinct partial recursive functions, then $\theta f$ and $\theta g$ are effectively inseparable.

Proof. Given $f, g$ we show there exists a recursive function $G$ of 2 variables such that if $\theta f \subseteq w_{i} \cap w_{j}^{\prime}, \theta g \subseteq w_{j} \cap w_{i}^{\prime}$, then $G(i, j) \in\left(w_{i} \cup w_{j}\right)^{\prime}$. 
Case 1. If $f \neq \varnothing, g \neq \varnothing$ the conclusion follows readily from Theorem 1 , by setting $G(i, j)=F(a, b, j, i)$ when $f=q_{a}, g=q_{b}$ since by hypothesis $w_{j} \subseteq \theta q_{a}^{\prime}$, $w_{i} \subseteq \theta q_{b}^{\prime}$.

Case 2. If either $f$ or $g=\varnothing$, the result is implied by the following:

Proposition 1. Let $f, g$ be partial recursive functions. Then there exist r.e. sets $\alpha, \beta$ with $\theta f \subseteq \alpha \cap \beta^{\prime}, \theta g \subseteq \beta \cap \alpha^{\prime}$ if and only if neither $f$ nor $g$ is an extension of the other.

Proof. This is a special case of Theorem 4.1 and 4.2 of [2], but a simple direct proof can be given:

First assume that neither $f$ nor $g$ extends the other. Then there exist $x_{0}, y_{0}$ such that $f\left(x_{0}\right)=y_{0}$ and either $g\left(x_{0}\right)$ is undefined or $g\left(x_{0}\right) \neq y_{0}$, and $x_{1}, y_{1}$ such that $g\left(x_{1}\right)=y_{1}$ and either $f\left(x_{1}\right)$ is undefined or $f\left(x_{1}\right) \neq y_{1}$. Then if $\alpha=\left\{n \mid q_{n}\left(x_{0}\right)=y_{0}\right\}$ and $\beta=\left\{n \mid q_{n}\left(x_{1}\right)=y_{1}\right\}$, we have $\theta f \subseteq \cap \beta^{\prime}$ and $\theta g \subseteq \beta \cap \alpha^{\prime}$, where $\alpha$ and $\beta$ are r.e. (In the usual way, $\alpha$ and $\beta$ can be replaced by disjoint r.e. sets $\gamma, \delta$ such that $\gamma \cup \delta=\alpha \cup \beta, \theta f \subseteq \gamma$ and $\theta g \subseteq \delta$.)

Now assume that $g$ is an extension of $f$, but that there exists an r.e. set $\alpha$ with $\theta f \subseteq \alpha, \theta g \subseteq \alpha^{\prime}$. Define a partial recursive function $G$ of 2 variables by

$$
G(n, x)=(\mu y)[y=f(x) \vee(n \in \alpha \wedge y=g(x))] .
$$

Since $g$ extends $f$, this is well-defined, and for fixed $n$ is a partial recursive function of $x$, so that $G(n, x)=q_{h(n)}(x)$ for a recursive $h$. Then by the second recursion theorem, there is an $e$ such that $q_{e}(x) \simeq q_{h(e)}(x)$. We now observe that $e \in \alpha$ implies $g(x) \simeq G(e, x) \simeq q_{h(e)}(x) \simeq q_{e}(x)$ so that $e \in \theta g \subseteq \alpha^{\prime}$, while $e \in \alpha^{\prime}$ implies

$$
f(x) \simeq G(e, x) \simeq q_{h(e)}(x) \simeq q_{e}(x)
$$

so that $e \in \theta f \subseteq \alpha$. Either way we obtain a contradiction.

To complete the proof of Corollary 1.1, note that if either $f$ or $g$ is null, the other extends it, so that any function $G$ will vacuously satisfy the conclusion.

Definition 2. In applications of Proposition 1, partial recursive functions $f$ and $g$, neither of which extends the other, will be called incomparable.

COROLlaRY 1.2. If $\alpha, \beta$ are distinct r.e. sets, $\theta \alpha$ and $\theta \beta$ are effectively inseparable.

Proof. Let $q_{a}, q_{b}$ be functions generating $\alpha, \beta$ respectively. Then since $\theta q_{a} \subseteq \theta \alpha$ and $\theta q_{b} \subseteq \theta \beta$, effective inseparability of the pair $\left(\theta q_{a}, \theta q_{b}\right)$ implies that of $(\theta \alpha, \theta \beta)$.

COROllary 1.3 (RICE). There are no nontrivial decidable classes of r.e. sets.

Proof. Let $A$ be a class of r.e. sets, $\theta A=\bigcup_{\alpha \in A} \theta \alpha$. Then if $\theta A$ is recursive, it follows that $\theta A$ and $\theta\left(A^{\prime}\right)=(\theta A)^{\prime}$ are separable by recursive sets, which by Corollary 1.2 implies that either $A$ or $A^{\prime}$ is void. 
DefinItion 3. A pair $(\alpha, \beta)$ of disjoint r.e. sets has doubly creative kernel $(\gamma, \delta)$ if $\gamma \subseteq \alpha, \delta \subseteq \beta$ and there is a recursive function $G$ of two variables such that whenever $w_{i} \subseteq \gamma^{\prime}$ and $w_{j} \subseteq \delta^{\prime}$, then $G(i, j) \in\left(\alpha \cup \beta \cup w_{i} \cup w_{j}\right)^{\prime}$.

THEOREM 2. If $q_{a}, q_{b}$ are distinct partial recursive functions, then the pairs $\left(w_{m}, w_{n}\right)$ of disjoint r.e. supersets of $\theta q_{a}, \theta q_{b}$ have doubly creative kernel $\left(\theta q_{a}, \theta q_{b}\right)$, uniformly in the following sense: there is a recursive function $G$ of six variables such that if $\theta q_{a} \subseteq w_{m}, \theta q_{b} \subseteq w_{n}, w_{m} \cap w_{n}=\varnothing$ and $w_{i} \subseteq \theta q_{a}^{\prime}, w_{j} \subseteq \theta q_{b}^{\prime}$, then $G(a, b, m, n, i, j) \in\left(w_{m} \cup w_{n} \cup w_{i} \cup w_{j}\right)^{\prime}$.

Proof. Let $f$ be a recursive function of two variables such that $w_{f(x, y)}=w_{x} \cup w_{y}$ for all $x, y$, and let $F$ be the recursive function of Theorem 1 ; then define a recursive function $G$ by $G(a, b, m, n, i, j)=F(a, b, f(n, i), f(m, j))$. The hypotheses then imply that $w_{f(n, i)} \subseteq \theta q_{a}^{\prime}, w_{f(m, j)} \subseteq \theta q_{b}^{\prime}$ and that $q_{a}$ and $q_{b}$ are distinct and non-null so that Theorem 1 applies and the conclusion follows.

COROLlaRY 2.1. If $f, g$ are incomparable partial recursive functions, then there exists a pair $(\alpha, \beta)$ of disjoint r.e. sets which has doubly creative kernel $(\theta f, \theta g)$.

Proof. By Proposition 1 and Theorem 2.

CoRollary 2.2. There exists a pair $(\gamma, \delta)$ of productive sets and a pair $(\alpha, \beta)$ of disjoint r.e. sets such that $(\alpha, \beta)$ has doubly creative kernel $(\gamma, \delta)$.

Proof. By Corollary 2.1, it suffices to show that $\theta f, \theta g$ are productive. This follows from Theorem 3.1 of [2].

It is shown in [10] that all doubly creative pairs of disjoint sets are doubly isomorphic under a recursive permutation of the non-negative integers. From this we obtain

THEOREM 3. Let $\alpha, \beta$ be disjoint r.e. sets. Then the following statements are equivalent:

(a) $(\alpha, \beta)$ is doubly creative,

(b) there is a pair of partial recursive functions $f, g$ and a recursive permutation $\pi$ such that $\pi(\theta f) \subseteq \alpha, \pi(\theta g) \subseteq \beta$,

(c) for every pair of incomparable functions $f, g$ there is a recursive permutation $\pi$ such that $\pi(\theta f) \subseteq \alpha$ and $\pi(\theta g) \subseteq \beta$.

Proof. (c) $\rightarrow$ (b) since there are pairs of incomparable functions.

(b) $\rightarrow$ (a): Let $f, g$ be a pair of partial recursive functions and assume that $\pi$ is a recursive permutation such that $\pi(\theta f) \subseteq \alpha$ and $\pi(\theta g) \subseteq \beta$. Then $\theta f \subseteq \pi^{-1}(\alpha), \theta g \subseteq \pi^{-1}(\beta)$, so that by Theorem $2, \pi^{-1}(\alpha)$ and $\pi^{-1}(\beta)$, which are disjoint r.e. sets, are doubly creative with kernel $(\theta f, \theta g)$. But this property is evidently a recursive isomorphism invariant, from which it follows that $(\alpha, \beta)$ is doubly creative with kernel $(\pi(\theta f), \pi(\theta g))$. 
(a) $\rightarrow$ (c): Assume $(\alpha, \beta)$ is doubly creative and let $f, g$ be incomparable functions. By Corollary 2.1, there exists a pair $(\gamma, \delta)$ of disjoint r.e. sets which is doubly creative with kernel $(\theta f, \theta g)$. Then by Smullyan's double isomorphism theorem, there is a recursive permutation $\pi$ such that $\alpha=\pi(\gamma) \supseteq \pi(\theta f)$ and $\beta=\pi(\delta) \supseteq \pi(\theta g)$.

THEOREM 4. An r.e. set $\alpha$ is creative if and only if for every pair $(f, g)$ of incomparable partial recursive functions there is an r.e. set $\beta$ and a recursive permutation $\pi$ such that $a \cap \beta=\varnothing$ and $(\alpha, \beta)$ is doubly creative with kernel $(\pi(\theta f), \pi(\theta g))$.

Proof. For one direction, it suffices to note that if $(\alpha, \beta)$ is doubly creative for some $\beta$, then $\alpha$ is creative. For the other direction, assume $\alpha$ is creative and that $f, g$ are incomparable functions. By Corollary 2.1, there is a pair $(\gamma, \delta)$ of disjoint r.e. sets with doubly creative kernel $(\theta f, \theta g)$. By Myhill's isomorphism theorem [5], there is a recursive permutation $\pi$ with $\alpha=\pi(\gamma)$; the conclusion follows by taking $\beta=\pi(\delta)$.

Note that Theorem 4 says nothing about the creativity of an r.e. set which may separate $\theta f$ from $\theta g$ in a case where these are not separable by a pair of r.e. sets. An argument similar to that of Theorem 1 gives, however, the following:

THEOREM 5. Let $q_{a}, q_{b}$ be partial recursive functions and assume $\alpha$ is an r.e. set such that $\theta q_{a} \subseteq \alpha, \theta q_{b} \subseteq \alpha^{\prime}$. Then $\alpha$ is creative (uniformly in $a, b$ and $a$ Gödel number of $\alpha$ ).

CoRollary 5.1. $(\theta \varnothing)^{\prime}$ is creative.

Proof. Apply Theorem 5 with $\alpha=(\theta \varnothing)^{\prime}, q_{b}=\varnothing$ and $q_{a}$ any non-null partial recursive function.

As one application of our results, we exhibit a class of partial recursive functions whose domains are creative, and which therefore have no general recursive extensions.

THEOREM 6. Let h be a partial recursive function. Define a functional $F$ by $F\left(q_{n}\right)=h(n)$ whenever defined. Denote by $E(F)$ the "extensional domain" of $F=\left\{f \mid\right.$ for all $m, n \in \theta f, F\left(q_{m}\right)$ and $F\left(q_{n}\right)$ are defined and $\left.F\left(q_{m}\right)=F\left(q_{n}\right)\right\}$. Then the domain of $h$ is a creative set unless $F$ is contant on $E(F)$.

Proof. Assume that $F$ is not constant on $E(F)$, i.e., that there exist $f, g \in E(F)$ and numbers $a, b$ such that $F(f)=a, F(g)=b, a \neq b$. Then $\theta f \subseteq \alpha=\{n \mid h(n)$ $=a\}$ while $\theta g \subseteq \beta=\{n \mid h(n)$ is defined but $h(n) \neq a\}$, where $\alpha$ and $\beta$ are r.e. and disjoint, so that by Theorem $3,(\alpha, \beta)$ is doubly creative. This implies that $\alpha \cup \beta=$ domain $h$ is creative; the same result evidently holds for all extensions of $h$.

Note that the hypothesis could be weakened as follows: Assume that there exist $f, g$ such that $h$ is defined (though not necessarily constant, nor even single- 
valued) on all of $\theta f$ and $\theta g$ in such a way that $h(\theta f)$ and $h(\theta g)$ are separable by recursive sets. Then domain $h$ is creative, by the same proof.

As an example, consider the function $v$ defined by Kleene ([4, pp. 346-347], $n=0$ ) with the property that if $w_{n} \neq \varnothing$, then $v(n) \in w_{n}$; evidently domain $v=\left\{m \mid w_{m} \neq \varnothing\right\}$ is creative. Theorem 6 then proves that this must be the case for every partial recursive extension $v^{*}$ of $v$. For let $A$ be the class of constant recursive functions, and define $F\left(q_{n}\right)=v^{*}(n)$; then $A \subseteq E(F)$ and $F$ is nonconstant on $A$.

Theorem 6 generalizes a property of partial recursive functionals $F$ whose domains (i.e., $\left\{n \mid F\left(q_{n}\right)\right.$ is defined $\}$ ) are "completely recursively enumerable" classes $\theta A$ which were shown in [2] to be creative (when $F$ is nonconstant). It constitutes a generalization in that not every functional $F$ with nonempty extensional domain (on which $F$ is an "effective operation") is potentially partial recursive [6].

It appears to us that it would be of some interest to investigate the possible isomorphism types of the doubly creative kernels of doubly creative pairs $\left({ }^{2}\right)$.

3. Relativized sets of indices of r.e. sets. The terminology of this section is that of [9] and [10]. Let $S$ be a consistent r.e. theory. An r.e. set $\alpha$ is representable in $S$ if there is a formula $A$ with one free variable such that for each non-negative integer $n, n \in \alpha$ implies $\vdash_{S} A(\bar{n})$, while $n \notin \alpha$ implies $\neg \vdash_{S} A(\bar{n})$, where $\bar{n}$ is the numeral in $S$ corresponding to $n$. If an r.e. set $\alpha$ is representable in $S$, we may relativize the notion of $\theta \alpha$ to the following:

Definition 4. $\theta_{S} \alpha=$ the set of Gödel numbers of formulas of $S$ containing one free variable which represent $\alpha$ in $S$.

It will be shown below that for many theories, if $\alpha$ and $\beta$ are (distinct) representable r.e. sets, then $\left(\theta_{s} \alpha, \theta_{S} \beta\right)$ is doubly isomorphic to $(\theta \alpha, \theta \beta)$, so that they possess the same recursively invariant properties, such as inducing double creativity on pairs of disjoint r.e. supersets. In the spirit of [1] and [9], however, we provide a direct proof of the latter property, which does not depend on the Myhill-Smullyan isomorphism theorems.

We require the following well-known result, whose proof we omit.

PROPOSITION 2. Let $S$ be a theory containing a formalization of the (primitive recursive) diagonal function $d(n)=$ the Gödel number of the formula obtained from the formula with Gödel number $n$ by replacing free $x$ by the numeral $\bar{n}$. Then for any formula $F(x, n)$ with two free variables we can effectively find a formula $W(n)$ with one free variable, whose Gödel number $w$ is recursive in that of $F(x, n)$, such that

$$
\vdash_{S} W(n) \equiv F(\bar{w}, n) .
$$

(2) For kernels $(\theta f, \theta g)$ where $f, g$ are incomparable functions, there are exactly 3 possible types. The proof will appear in Proc. Amer. Math. Soc. 
THEOREM 5. Let $S$ be a consistent r.e. theory containing the diagonal function. Assume that $\sigma, \pi, \alpha, \beta$ are r.e. sets such that $\sigma$ and $\pi$ are representable in $S$ by formulas $R$ and $P$ respectively, that $\theta_{S} \pi \subseteq \alpha, \theta_{S} \sigma \subseteq \beta$, and that $\alpha, \beta$ are strongly separable in $S$ (i.e., there is a formula A such that $n \in \alpha$ implies $\vdash_{s} A(\bar{n})$ and $n \in \beta$ implies $\vdash_{S} \neg A(\bar{n})$ ). Then there is a number $w$, which can be effectively found from the Gödel numbers of $R, P$ and $A$, such that $w \in(\alpha \cup \beta)^{\prime}$.

Proof. Let $F(x, n)$ be $A(x) \supset R(n) \cdot \wedge \cdot \neg A(x) \supset P(n)$. By Proposition 2, there is a $w$ recursive in the Gödel number of $F$ and thus in those of $A, R, P$, such that $r_{s} W(n) \equiv F(\bar{w}, n)$. Now $r_{s} A(x) \cdot \supset \cdot F(x, n) \equiv R(n)$ and

$$
\left.\vdash_{S}\right\urcorner A(x) \cdot \supset \cdot F(x, n) \equiv P(n) \text {. }
$$

We claim that $w \in(\alpha \cup \beta)^{\prime}$. Assume not:

Case 1. $w \in \alpha$. Then $\vdash_{s} A(\bar{w})$ which implies $\vdash_{S} F(\bar{w}, n) \equiv R(n)$. So $\vdash_{s} W(n)$ $\equiv R(n)$, which implies that $W(n)$ represents $\sigma$ in $S$ and $w \in \theta_{S} \sigma \subseteq \beta$. Then $\vdash_{S} \neg A(\bar{w})$, and $S$ is inconsistent.

Case 2. $w \in \beta$. Then $\vdash_{s} \neg A(\bar{w})$ which implies $\vdash_{S} F(\bar{w}, n) \equiv P(n)$. So $\vdash_{S} W(n) \equiv P(n)$, which implies that $w \in \theta_{S} \pi \subseteq \alpha$. Then $\vdash_{S} A(w)$, which again contradicts the hypothesis that $S$ is consistent.

COROLlary 5.1. Let $S$ be a consistent r.e. theory in which all r.e. sets are effectively representable and pairs of disjoint r.e. sets are effectively (strongly) separable. Then there is a recursive function $F$ of six variables such that if $\theta_{S} w_{a} \subseteq w_{m} \cap w_{n}^{\prime}, \theta_{S} w_{b} \subseteq w_{n} \cap w_{m}^{\prime}$ then $F(a, b, m, n, i, j)$ is a doubly creative function for $\left(w_{m}, w_{n}\right)$ (i.e., a doubly productive function for $\left.\left(w_{m}^{\prime}, w_{n}^{\prime}\right)\right)$.

Proof. Assume that $w_{i} \subseteq w_{m}^{\prime}, w_{j} \subseteq w_{n}^{\prime}$. Let $w_{c}=\left\{x \mid x\right.$ appears in $w_{m} \cup w_{j}$ before appearing in $w_{n} \cup w_{i}$ in a simultaneous enumeration $\}, w_{d}=\{x \mid x$ appears in $w_{n} \cup w_{i}$ before appearing in $w_{m} \cup W_{j}$ ). (This can be formalized as in the proof of Theorem 1.) Then $w_{c} \cap w_{d}=\varnothing, \theta_{S} w_{a} \subseteq w_{c}, \theta_{S} w_{b} \subseteq w_{d}$. Setting $\alpha=w_{c}, \beta=w_{d}$, $\pi=w_{a}$ and $\sigma=w_{b}$ in Theorem 5, we observe that since according to the hypothesis we can effectively compute the Gödel numbers of $R$ and $P$ from $a$ and $b$ and that of $\boldsymbol{A}$ from $c$ and $d$ and thus from $m, n, i, j$, then we can effectively find a number $w \in\left(w_{m} \cup w_{n} \cup w_{i} \cup w_{j}\right)^{\prime}$. Then set $F(a, b, m, n, i, j)=w$.

To add significance to these results, we note that by the results of [3], [8], [9], the hypothesis of effective representability and separability holds in ordinary theories of arithmetic (in which recursive functions are definable and which contain a " $\leqq$ " relation with the usual properties).

Isomorphism results. For the following, recall that $\alpha$ is 1-1 reducible to $\beta\left(\alpha R_{1} \beta\right)$ if there is a 1-1 recursive function $f$ such that $x \in \alpha \leftrightarrow f(x) \in \beta$. We say that $f$ reduces $\alpha$ to $\beta$ and that $\alpha \equiv_{1} \beta$ if $\alpha R_{1} \beta$ and $\beta R_{1} \alpha$.

Let $S$ be an "effective Gödel theory" in the sense of [10], i.e., a theory for 
which there is a recursive function $g$ such that the formula with Gödel number (g. n.) $g(i), F_{g(i)}$, represents $w_{i}$ in $S$. Let $T$ denote the set of Gödel numbers of provable sentences of $S, R$ the set of Gödel numbers of refutable sentences of $S$.

(1) $\theta w_{i} \equiv_{1} \theta_{S} w_{i}$, uniformly in $i$; i.e., there are recursive functions $h, k$ such that $h(x)$ reduces $\theta w_{i}$ 1-1 to $\theta_{S} w_{i}$ and $k(x)$ reduces $\theta_{S} w_{i} 1-1$ to $\theta w_{i}$, for all $i$.

Proof. Define $h$ by setting $w_{h(n)}=\left\{m \mid{ }_{s} F_{n}(\bar{m})\right\}$, which is an r.e. set since $S$ is an r.e. theory. Then $h$ is a recursive function which can be taken to be 1-1 by the iteration theorem, and $n \in \theta_{S} w_{i} \leftrightarrow F_{n}$ represents $w_{i}$ in $S \leftrightarrow w_{i}=w_{h(n)} \leftrightarrow h(n) \in \theta w_{i}$. So $h$ reduces $\theta_{s} w_{i}$ 1-1 to $\theta w_{i}$.

Now let $x_{1}, x_{2}, \cdots$ be distinct variables of $S$, and let $F_{g(n)}$ represent $w_{n}$ in $S$. Define $k$ by setting $k(n)=g . n .\left(F_{g(n)}(x) \wedge x_{n}=x_{n}\right)$. Then $k$ is a 1-1 recursive function, and $\vdash_{S} F_{g(n)}(\bar{m})$ if and only if $\vdash_{S} F_{k(n)}(\bar{m})$; this implies $n \in \theta w_{i} \leftrightarrow w_{n}$ $=w_{i} \leftrightarrow F_{g(n)}$ represents $w_{i}$ in $S \leftrightarrow F_{k(n)}$ represents $w_{i}$ in $S \leftrightarrow k(n) \in \theta_{S} w_{i}$. So $k$ reduces $\theta w_{i}$ 1-1 to $\theta_{s} w_{i}$.

(2) We conclude from (1) and Proposition 10 of [10] that there is a recursive permutation $\pi$ such that $\pi$ is an isomorphism between $\theta w_{i}$ and $\theta_{S} w_{i}$ for all $i$. Thus all recursively invariant properties of sets $\theta w_{i}$ and pairs $\left(\theta w_{i}, \theta w_{j}\right)$ carry over to $\theta_{S} w_{i}$ and $\left(\theta_{S} w_{i}, \theta_{S} w_{j}\right)$.

(3) It now follows that if $S_{1}$ and $S_{2}$ are theories such that

(i) the set of g.n.'s of formulas with one free variable is recursive,

(ii) pairs of disjoint r.e. sets are strongly separable,

(iii) all r.e. sets are effectively representable, then there is a recursive isomorphism $\pi$ under which $T_{1}$ corresponds to $T_{2}, R_{1}$ to $R_{2}$ and $\theta_{S_{1}} w_{i}$ to $\theta_{S_{2}} w_{i}$ for each $i$.

Proof. By (2), there are recursive isomorphisms $\pi_{1}$ and $\pi_{2}$ such that $\pi_{j}\left(\theta w_{i}\right)=\theta_{S_{j}} w_{i}$ for each $i$, for $j=1,2$; then $\pi_{3}=\pi_{2} \pi_{1}^{-1}$ is a recursive isomorphism mapping $\theta_{S_{1}} w_{i}$ onto $\theta_{S_{2}} w_{i}$ for each $i$. By Theorem 28 of [10], there is a recursive isomorphism $\pi_{4}$ mapping $T_{1}$ onto $T_{2}$ and $R_{1}$ onto $R_{2}$. Now $n \in \bigcup_{i=0}^{\infty} \theta_{S} w_{i}$ if and only if $n$ is the Gödel number of a formula containing one free variable, and by assumption, $\beta=\bigcup_{i=0}^{\infty} \theta_{S_{1}} w_{i}$ is a recursive set disjoint from $T_{1} \cup R_{1}$. Thus it suffices to define $\pi=\pi_{3}$ on $\beta, \pi=\pi_{4}$ on $\beta^{\prime}$.

\section{REFERENCES}

1. P. Bernays, Review of Creative sets by J. Myhill [5], J. Symbolic Logic 22 (1957), 73-76.

2. J. C. E. Dekker and J. Myhill, Some theorems of classes of recursively enumerable sets, Trans. Amer. Math. Soc. 89 (1958), 25-59.

3. A. Ehrenfeucht and $\mathrm{S}$. Feferman, Representability of recursively enumerable sets in formal theories, Arch. Math. Logik Grundlagenforsch. 5 (1960), 37-41.

4. S. C. Kleene, Introduction to metamathematics, Van Nostrand, New York, 1952.

5. J. Myhill, Creative sets, Z. Math. Logik Grundlagen Math. 1 (1955), 97-108.

6. G. Kreisel, D. Lacombe and J. R. Shoenfield, Partial recursive functionals and effective operations, Constructivity in mathematics: Proceedings of the colloquium held at Amsterdam, 1957. 
7. E. L. Post, Recursively enumerable sets of positive integers and their decision problems, Bull. Amer. Math. Soc. 50 (1944), 284-316.

8. H. Putnam and R. M. Smullyan, Exact separation of recursively enumerable sets within theories, Proc. Amer. Math. Soc. 11 (1960), 574-577.

9. J. C. Shepherdson, Representability of recursively enumerable sets in formal theories, Arch. Math. Logik Grundlagenforsch. 5.(1961), 119-127.

10. R. M. Smullyan, Theory of formal systems, Annals of Mathematics Studies, No. 47, Princeton Univ. Press, Princeton, N. J., 1961.

Mount Holyoke College,

South Hadley, Massachusetts 